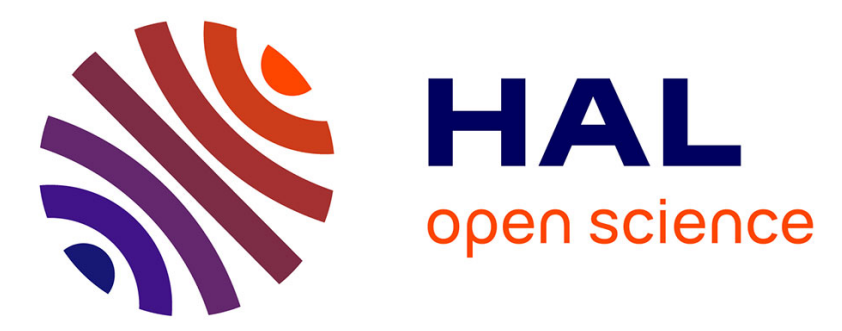

\title{
Post-buckling evolution of wavy patterns in trapezoidal film/substrate bilayers
}

\author{
Fan Xu, Ting Wang, Chenbo Fu, Yu Cong, Yongzhong Huo, Michel
} Potier-Ferry

\section{- To cite this version:}

Fan Xu, Ting Wang, Chenbo Fu, Yu Cong, Yongzhong Huo, et al.. Post-buckling evolution of wavy patterns in trapezoidal film/substrate bilayers. International Journal of Non-Linear Mechanics, 2017, 96, pp.46-55. 10.1016/j.ijnonlinmec.2017.08.006 . hal-01592437

\section{HAL Id: hal-01592437 \\ https://hal.science/hal-01592437}

Submitted on 24 Sep 2017

HAL is a multi-disciplinary open access archive for the deposit and dissemination of scientific research documents, whether they are published or not. The documents may come from teaching and research institutions in France or abroad, or from public or private research centers.
L'archive ouverte pluridisciplinaire HAL, est destinée au dépôt et à la diffusion de documents scientifiques de niveau recherche, publiés ou non, émanant des établissements d'enseignement et de recherche français ou étrangers, des laboratoires publics ou privés. 


\title{
Post-buckling evolution of wavy patterns in trapezoidal film/substrate bilayers
}

\author{
F. Xu ${ }^{\text {a }}$, T. Wang ${ }^{\text {a }}$, C. Fu ${ }^{\text {a }}$, Y. Cong ${ }^{\mathrm{b}}$, Y. Huo ${ }^{\mathrm{a}}$, M. Potier-Ferry ${ }^{\mathrm{c}}$ \\ a Institute of Mechanics and Computational Engineering, Department of Aeronautics and Astronautics, Fudan University, 220 Handan Road, Shanghai 200433, PR China \\ b ESI Group, R\&D, 99 Rue des Solets, 94513 Rungis, France \\ ' Laboratoire d'Etude des Microstructures et de Mécanique des Matériaux, LEM3, UMR CNRS 7239, Université de Lorraine, 7 Rue Félix Savart, BP 15082, 57073 Metz \\ Cedex 03, France
}

This paper investigates quantitatively the post-buckling response of irregular wrinkles in a trapezoidal film/substrate bilayer. The geometric gradient can change the wrinkling profile to create ribbed and graded structural patterns with variations of wave direction, amplitude and wavelength. The tapered angle and edge dimension are examined numerically using a nonlinear shell/solid coupled finite element model that incorporates a path-following continuation technique, which explores their influences on secondary bifurcations, localization and surface mode transition. For instance, the competition between plate-like and beam-like post-buckling behavior is discussed. An analysis of graded amplitude is also provided based on Fourier envelope equations of beam/foundation models, which gives an insightful understanding of these fading wrinkles that differ from the ones usually observed in rectangular geometric cases. The results of this work can be used to guide the design of geometrically gradient film/substrate systems to achieve desired wavy instability patterns.
Keywords: Graded wrinkling Trapezoidal film/substrate Post-buckling Bifurcation

\section{Introduction}

Quantitative understanding and prediction of wrinkling pattern formation and evolution is of great importance not only for comprehensive knowledge on morphogenesis of growing soft tissues widely observed in nature and biological systems [1-7], but also for large industrial application areas including functional surface patterning design [8,9], micro/nano-fabrication of flexible electronic devices [10,11], microlens arrays production [12], adaptive aerodynamic drag control [13,14], mechanical property measurement of material characteristics [15], and the design of moisture-responsive wrinkling devices with tunable dynamics [16] and reversible optical writing/erasure functional surface [17]. Previous works are mainly concerned with 2D or 3D rectangular film/substrate systems and suggest that wrinkling patterns strongly depend on applied loading [18-28]. For instance, a 3D rectangular film/substrate structure usually exhibits uniform sinusoidal wrinkling patterns under uniaxial compression, except in the boundary layers where stress concentration may occur and lead to localization mode [23]. A recent investigation [29] demonstrates that graded wrinkles with varying amplitude and wavelength can appear in gradient structured topology, e.g. trapezoidal geometry, which suggests that the stress gradient stemming from irregular geometric topology can change wrinkling profile to create ribbed and graded structural texture, for example shark skin is covered with ribbed, graded texture aligned in the streamwise direction. Critical buckling conditions including load, mode shape and wavelength can be deduced through linearized eigenvalue analyses. Such finite element simulations have been performed by Raayai-Ardakani et al. [29], but bifurcation analysis is still missing. Therefore, a systematic study on how irregular post-buckling patterns quantitatively evolve and their associated instability modes in trapezoidal geometry still merits further efforts.

This work aims at exploring the occurrence and post-bifurcation evolution of irregular wrinkling patterns in uniaxially compressed trapezoidal film/substrate structures through advanced numerical methods. A beam/foundation model [30] and a 3D shell/solid coupled finite element model [23] are respectively applied to quantitatively predict spatial surface morphology. The models incorporate Asymptotic Numerical Method (ANM) [31] which is a robust path-following continuation technique to trace secondary bifurcations on their post-buckling evolution path, i.e. bifurcation diagram, as the load increases. We consider geometrically perfect film/substrate system without any imperfection.

\footnotetext{
* Corresponding author.

E-mail address: fanxu@fudan.edu.cn (F. Xu).
} 


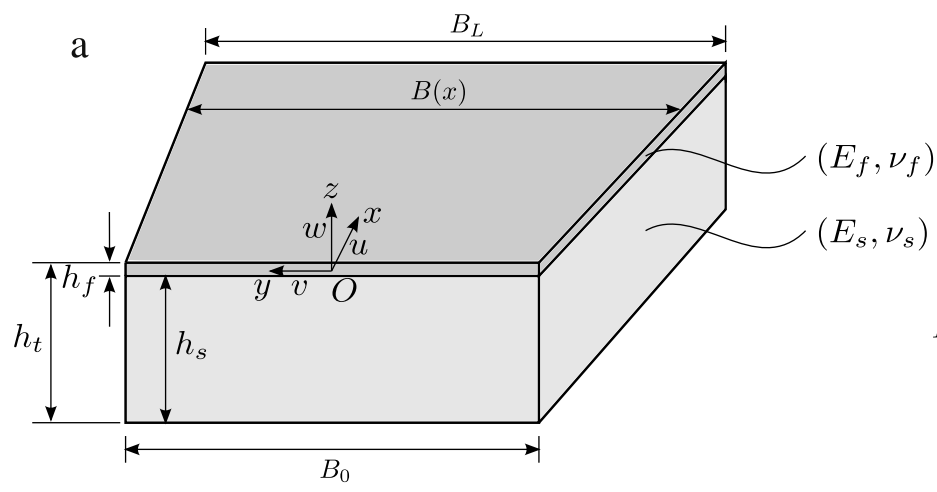

b

Fig. 1. Geometry of trapezoidal film/substrate system: (a) Airview; (b) Top view.

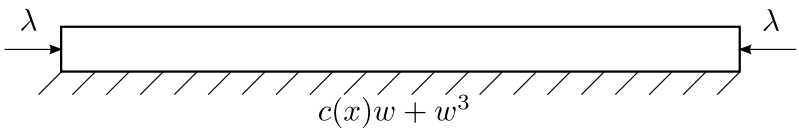

Fig. 2. Sketch of an elastic beam attached on a nonlinear elastic foundation.

The paper is organized as follows. In Section 2, a theoretical analysis based on a nonlinear beam/foundation equation is recalled to find critical buckling parameters. Then an analysis of graded amplitude is provided in Section 3, based on a microscopic beam/foundation model and a Fourier-related envelope model. In Section 4, a 3D nonlinear shell/solid finite element model [23] is employed for post-buckling analyses of 3D trapezoidal film/substrate systems. Concluding remarks are given in Section 5.

\section{Sinusoidal wrinkling with geometric gradient effect}

We consider an elastic thin film bonded to a soft substrate under uniaxial compression $F$, in the case of linearly tapered geometry with an angle $\theta$, as shown in Fig. 1. The geometric gradient leads to stress gradient that can alter uniform sinusoidal wrinkles to graded undulations, where the amplitude, wavelength and direction may vary along the length simultaneously. Upon wrinkling, the film elastically buckles to relax the compressive stress, and the substrate concurrently deforms to maintain perfect bonding at the interface. The potential energy of the system is considered within the framework of Hookean elasticity, since the macroscopic strain is usually limited to linear regime and will not exceed $4 \%$, by taking the large modulus ratio $E_{f} / E_{s} \geqslant \mathcal{O}\left(10^{2}\right)$ into account [29,32]. Nevertheless, in the post-buckling stage, the film can undergo geometrically nonlinear rotations and displacements under excess compression.

Let $x$ and $y$ be in-plane coordinates, while $z$ is the direction perpendicular to the mean plane of the film/substrate. The length of the system is denoted by $L$, and the shorter and longer widths are represented by $B_{0}$ and $B_{L}$, respectively. The parameters $h_{f}, h_{s}$ and $h_{t}$ represent, respectively, the thickness of the film, the substrate and the total thickness of the system. Young's modulus and Poisson's ratio of the film are respectively denoted by $E_{f}$ and $v_{f}$, while $E_{s}$ and $v_{s}$ are the corresponding material properties for the substrate.

The geometric gradient generates non-uniform distributed axial stress $\sigma_{f}(x)$ in the film:

$\sigma_{f}(x)=\frac{F}{h_{f} B(x)}$,

where the width of the system $B(x)$ is given as

$B(x)=B_{L}-2(L-x) \tan \theta$.

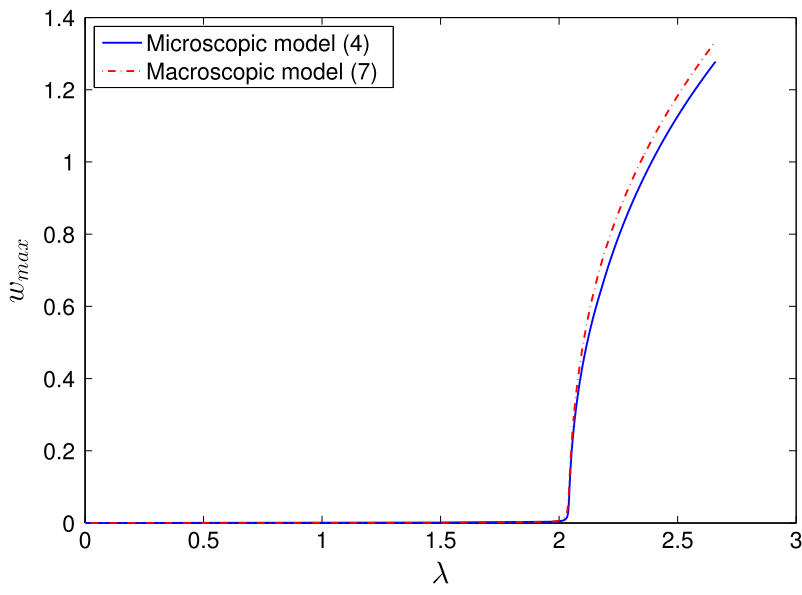

Fig. 3. Bifurcation curves of the microscopic model (4) and macroscopic model (7). The analytical critical load $\lambda_{c r}=2$ agrees with the numerical solutions.

For 1D sinusoidal wrinkles, nonlinear Föppl-von Kármán plate equations that govern the deflection of the film can be reduced to a fourth-order ordinary differential equation (ODE), namely a nonlinear beam model, which can be solved from eigenvalue analysis where the critical load $F_{c r}$ and wavelength $\ell_{c r}$ are obtained by minimizing the corresponding parameters with respect to eigenvalues [29]:

$\left\{\begin{array}{l}\frac{F_{c r}}{B_{L}}=\frac{E_{f} h_{f}}{4}\left(\frac{3 E_{s}}{E_{f}}\right)^{2 / 3}\left(\frac{2 L \tan \theta}{B_{0} \ln \left(B_{L} / B_{0}\right)}\right), \\ \frac{\ell_{c r}}{2 \pi h_{f}}=\left(\frac{E_{f}}{3 E_{s}}\right)^{1 / 3}\left(\frac{B_{L} \ln \left(B_{L} / B_{0}\right)}{2 L \tan \theta}\right)^{1 / 2},\end{array}\right.$

where the last terms on the right-hand sides demonstrate the effect of tapered geometry compared with rectangular geometry $[18,19]$.

Even though the eigenvalue analysis captures the onset of instabilities, nonlinear buckling analyses are essential to determine the postbuckling response and the mode transition of surface wrinkles. Besides, deformations obtained from eigenvalue analysis are eigenvectors rather than the actual deformation itself [33]. Thus, in what follows, postbuckling analyses will be based either on a nonlinear beam/foundation model or on a shell/solid coupled 3D finite element model. The resulting differential equations will be solved by a path-following continuation technique which is able to quantitatively trace the post-bifurcation evolution.

\section{Microscopic and macroscopic graded wrinkling}

To investigate 1D graded wrinkles, we recall a classical example of an elastic beam lying on a nonlinear elastic foundation, as shown in Fig. 2. 

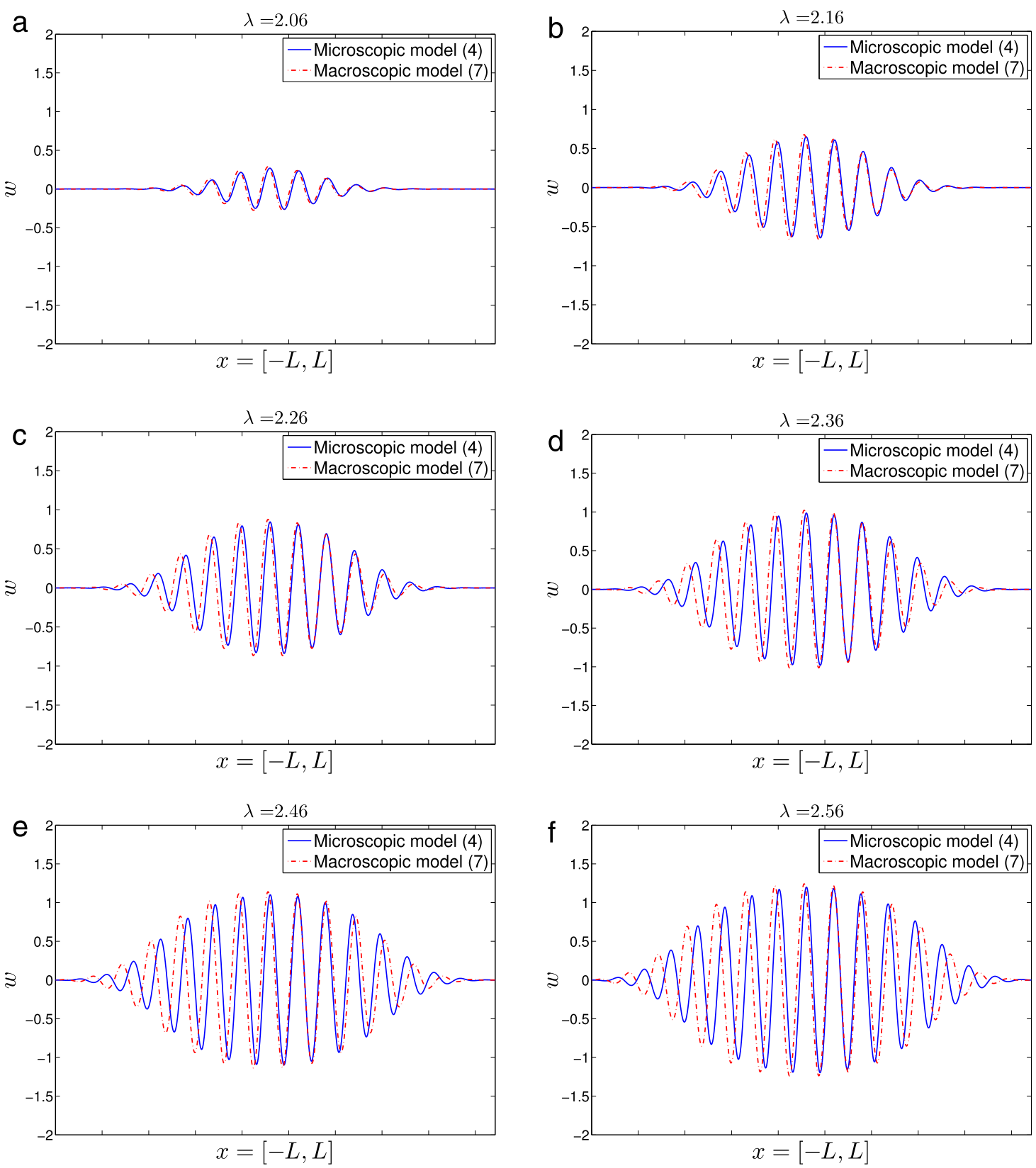

Fig. 4. Wrinkling evolution of the microscopic model (4) and macroscopic model (7) with an increasing load from (a) to (f).
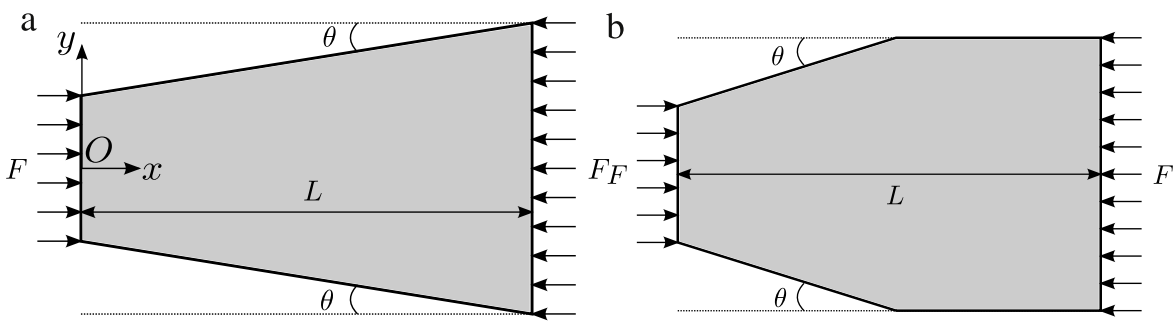

Fig. 5. Film/substrate subjected to uniaxial compression: (a) Trapezoidal film/substrate (Film/Sub I-III); (b) Trapezoidal-rectangular film/substrate (Film/Sub IV-VII).

The unknown is deflection $w(x)$ and the problem depends on a scalar parameter $\lambda$ which represents the applied compressive load. The governing ODE which is similar to the Swift-Hohenberg equation reads $\frac{\mathrm{d}^{4} w}{\mathrm{~d} x^{4}}+\lambda \frac{\mathrm{d}^{2} w}{\mathrm{~d} x^{2}}+c(x) w+w^{3}=0$.

This film/substrate system is able to describe periodic wrinkles. For instance, with a constant stiffness of foundation $c$, relation between the 


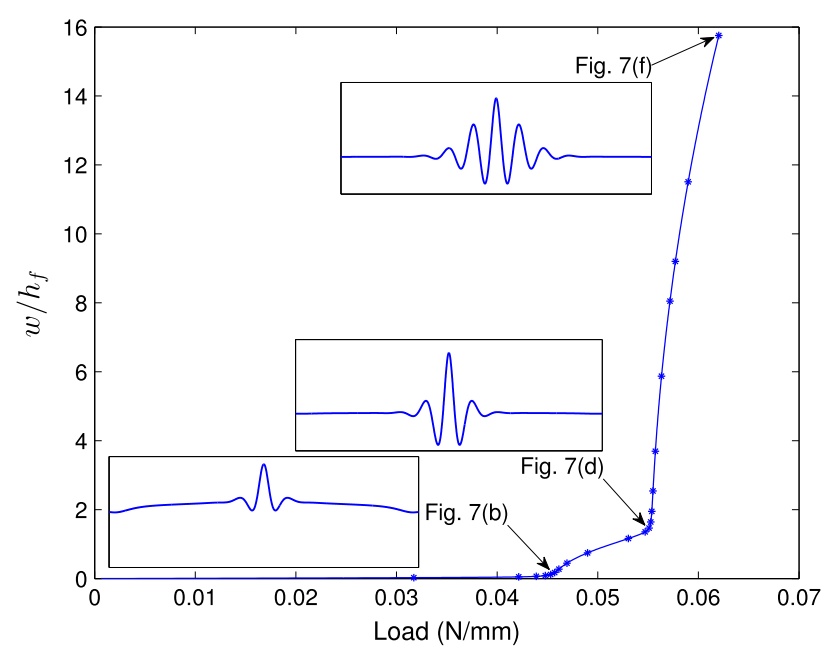

Fig. 6. Bifurcation curve of Film/Sub I with $\tan \theta=0.25, L=1 \mathrm{~mm}$ and $B_{0}=0.75 \mathrm{~mm}$. Representative wrinkling shapes in Fig. 7 on the post-buckling evolution path are marked. Each point corresponds to one incremental step. critical load $\lambda$ and wave number $q$ of periodic patterns can be deduced from the linearized version of (4):

$$
\lambda(q)=q^{2}+\frac{c}{q^{2}} .
$$

The critical wave number $q=\sqrt[4]{c}$ and critical load $\lambda_{c r}=\min \lambda(q)=$ $2 \sqrt{c}=2 q^{2}=2 c / q^{2}$ can be defined as the minimum of the neutral stability curve $\lambda(q)$.

To explore graded wrinkles, we further consider a variable foundation stiffness $c(x)$ as follows:

$c(x)=1+a\left(\frac{x}{L}\right)^{2}, \quad x \in[-L, L]$,

where $a$ is a positive constant representing the spatial variation of foundation stiffness. Thus, the critical load varies with the minimum value in the middle $\left(\lambda_{c r}=2\right)$ and maximum value on the boundary $\left(\lambda_{c r}=2 \sqrt{1+a}\right)$, which means that the instability first occurs in the center and then propagate progressively to the boundary.

We can then write the corresponding Fourier-related macroscopic form of the microscopic model (4) through introducing an envelope $w_{1}(x)$, by ensuring $w(x)=w_{1}(x) \exp (i q x)+\bar{w}_{1} \exp (-i q x)$ or $w(x)=$ $2 w_{1}(x) \sin (q x)$ in the case where the envelope $w_{1}(x)$ is real valued
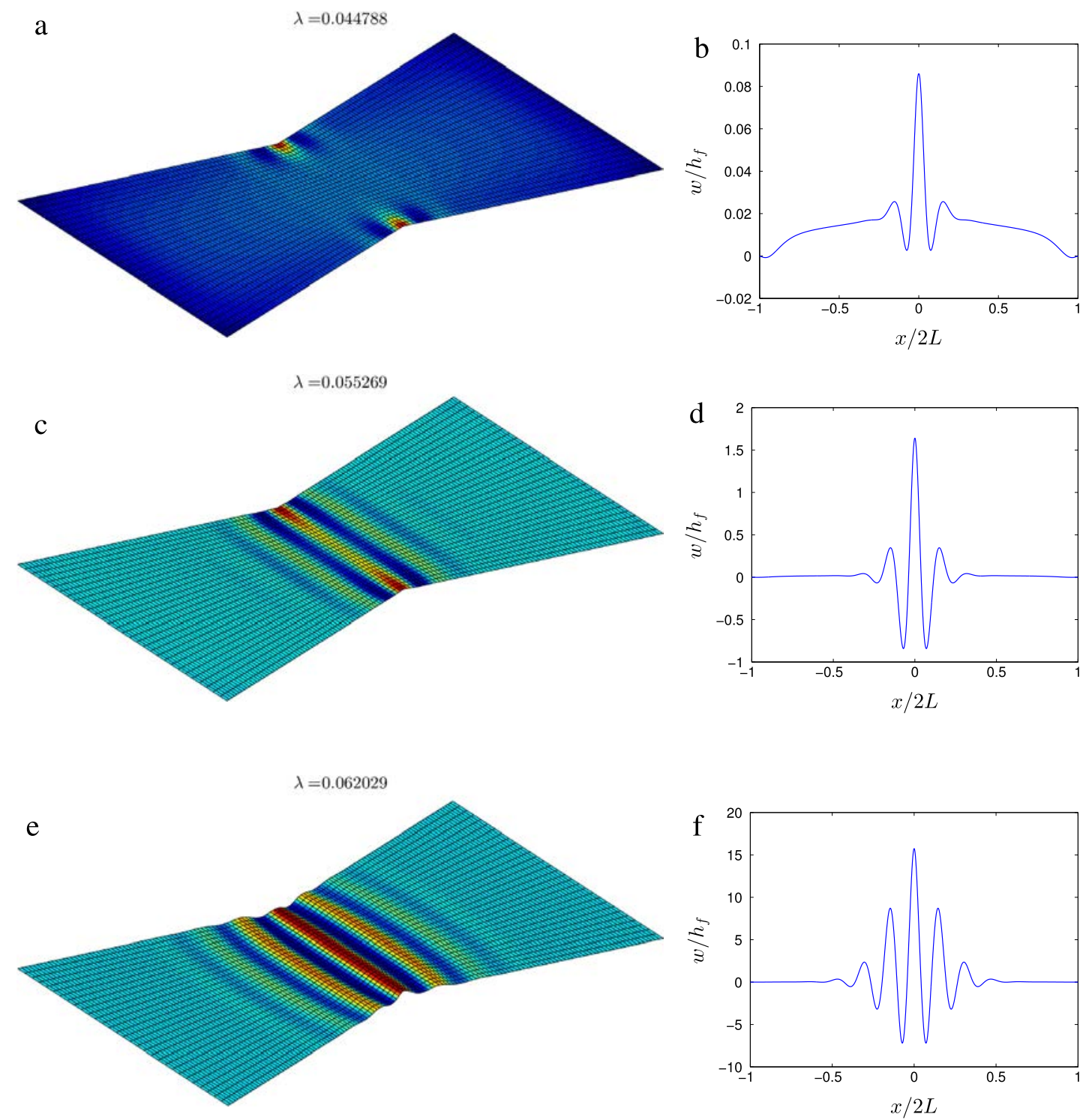

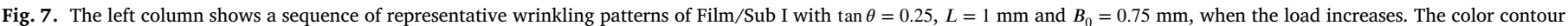
represents $w / h_{f}$. The right column presents the corresponding wrinkling modes along the length. 


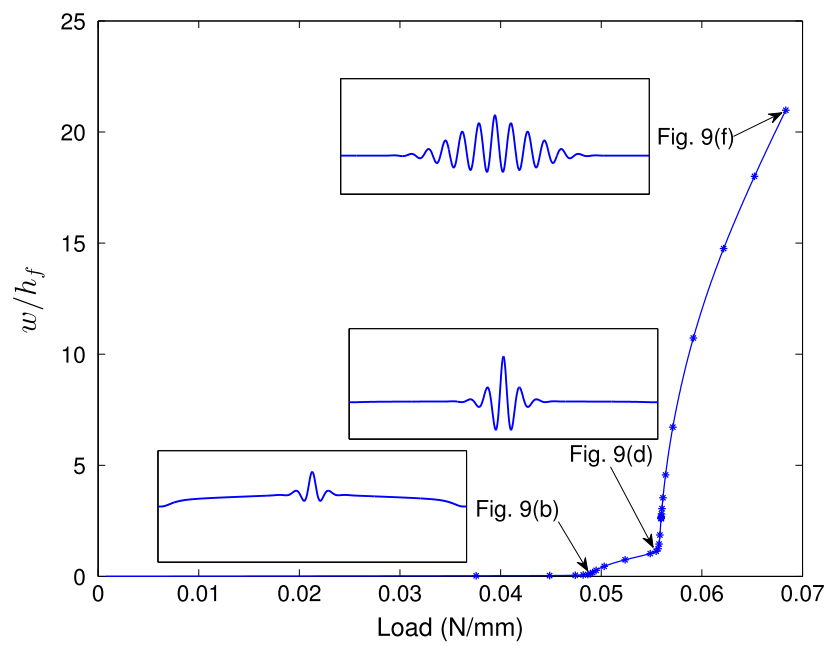

Fig. 8. Bifurcation curve of Film/Sub II with $\tan \theta=0.15, L=1.5 \mathrm{~mm}$ and $B_{0}=0.75 \mathrm{~mm}$. Representative wrinkling shapes in Fig. 9 on the post-buckling evolution path are marked. Each point corresponds to one incremental step. (see [30] for more details):

$-(6-\lambda) \frac{\mathrm{d}^{2} w_{1}}{\mathrm{~d} x^{2}}+[1+c(x)-\lambda] w_{1}+3 w_{1}^{3}=0$.

This envelope equation (7) can describe both the collective behavior of wrinkles and local appearance of instability patterns. The local character of the instability can be seen by disregarding the first term of (7):

$[1+c(x)-\lambda] w_{1}+3 w_{1}^{3}=0$.

This equation was referred in the instability community [34] as Landau equation, while the full envelope equation (7) is Ginzburg-Landau equation with a spatially variable coefficient $c(x)$. According to the Landau model, a classical pitchfork bifurcation can be defined at each point $x$, with local critical load and local value of the envelope being:

$\left\{\begin{array}{l}\lambda_{l o c}=1+c(x), \\ w_{l o c}= \pm \sqrt{\frac{\lambda_{l o c}(x)-\lambda}{3}} .\end{array}\right.$

This explains clearly why instability appears first in the region with the weakest stiffness $c(x)$, here in the middle. According to (9), amplitude follows the same distribution. The same qualitative results should remain valid for trapezoidal systems, with local stiffness being inversely proportional to the width of the film/substrate system.
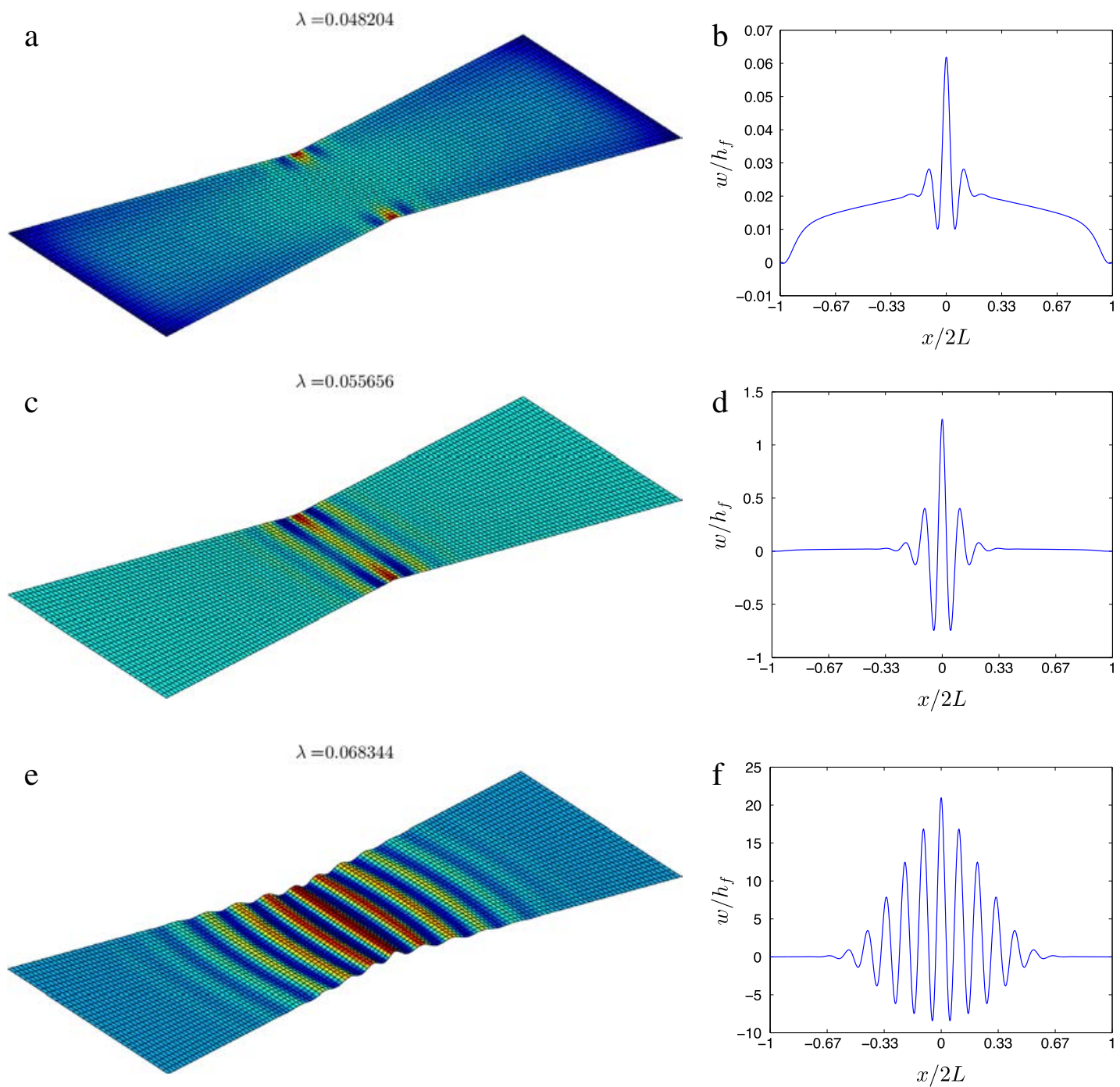

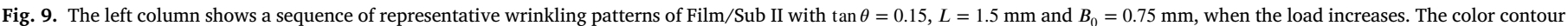
represents $w / h_{f}$. The right column presents the corresponding wrinkling modes along the length. 


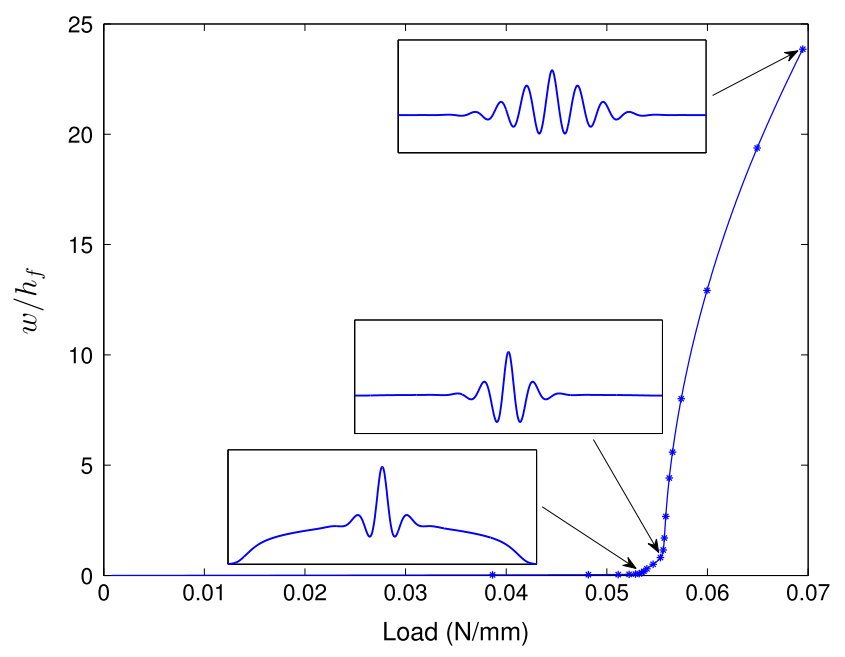

Fig. 10. Bifurcation curve of Film/Sub III with $\tan \theta=0.15, L=1 \mathrm{~mm}$ and $B_{0}=0.375$ $\mathrm{mm}$. Each point corresponds to one incremental step.

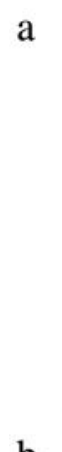

b
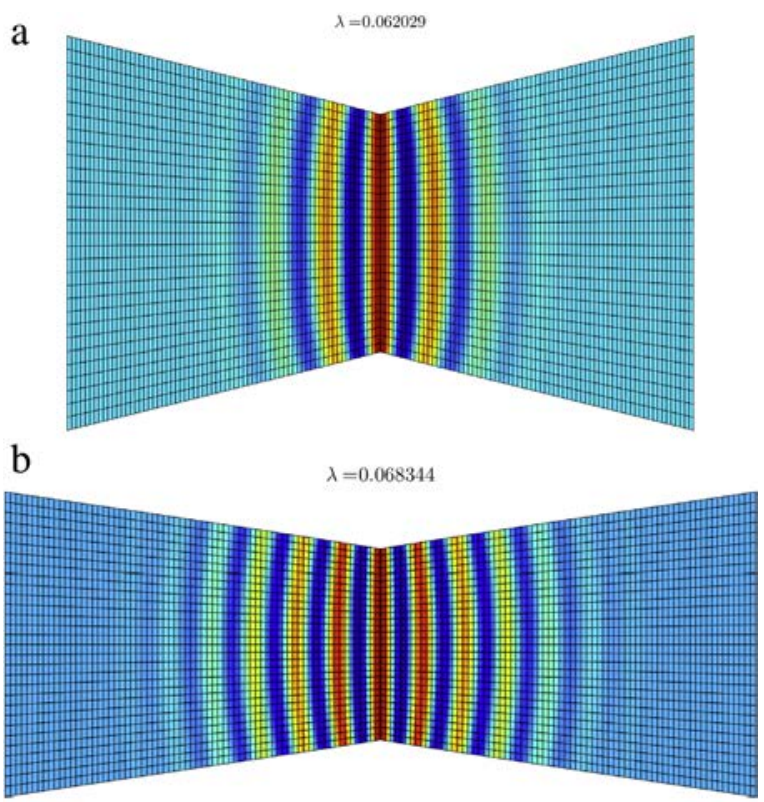

C

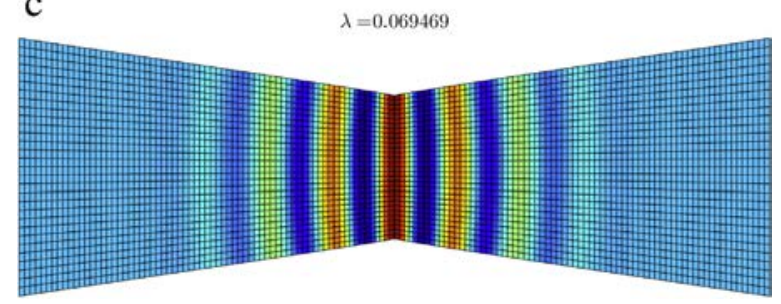

Fig. 11. Top view of final patterns: (a) Film/Sub I; (b) Film/Sub II; (c) Film/Sub III. The color contour denotes $w / h_{f}$.

Table 1

Common material characteristics and geometric properties.

\begin{tabular}{llllll}
\hline$E_{f}(\mathrm{MPa})$ & $E_{s}(\mathrm{MPa})$ & $v_{f}$ & $v_{s}$ & $h_{f}(\mathrm{~mm})$ & $h_{s}(\mathrm{~mm})$ \\
\hline $1.3 \times 10^{5}$ & 1.8 & 0.3 & 0.48 & $10^{-3}$ & 0.1 \\
\hline
\end{tabular}

Both ODEs (4) and (7) can be solved numerically by finite element method to investigate post-buckling pattern evolution [30], with given values of $a=1$ and $L=15 \pi$, for example. Load-displacement curves
Table 2

Different dimensional parameters.

\begin{tabular}{lllll}
\hline Film/Sub & $L(\mathrm{~mm})$ & $B_{0}(\mathrm{~mm})$ & $B_{L}(\mathrm{~mm})$ & $\tan \theta$ \\
\hline I & 1 & 0.75 & 1.25 & 0.25 \\
II & 1.5 & 0.75 & 1.2 & 0.15 \\
III & 1 & 0.375 & 0.675 & 0.15 \\
IV & 1.2 & 0.8 & 1.2 & 0.25 \\
V & 1.2 & 0.8 & 1.2 & 0.5 \\
VI & 1.2 & 0.4 & 1.2 & 0.5 \\
VII & 1.2 & 0.4 & 1.2 & 1 \\
\hline
\end{tabular}

are depicted in Fig. 3 and the evolution of graded wrinkles is presented in Fig. 4, where the critical load corresponds to the analytical solution $\lambda_{c r}=2$. Precisely, wrinkles first appear in the center with the minimum stiffness and then propagate gradually to the boundary. This graded wrinkling depends on the variable coefficient in the envelope equation (7) and can also be described by the microscopic model (4). Phase difference between microscopic and macroscopic models is observed. This discrepancy is mainly because the simplest macroscopic model (7) with real envelopes cannot account for phase variation near the boundary. There exist other Ginzburg-Landau equations with complex envelopes which could explain this phase evolution [35-37].

\section{Finite element results and discussion}

Spatially graded wrinkles and their post-buckling evolution for uniaxially compressed trapezoidal film/substrate structures will be investigated numerically through the established 3D finite element model, which was first introduced by Xu et al. [23] and subsequently applied to hyperelastic film/substrate [32]. The models incorporate a pathfollowing continuation technique named ANM [31]. It can be applied to solve nonlinear partial differential equations, and appears as an efficient continuation predictor which does not require corrective iterations to trace post-buckling evolution on the equilibrium path and to predict secondary bifurcations without using special tool. This finite element framework is sufficiently versatile for the present case of linearly tapered geometry. In this model, the surface layer is represented by a thin shell model to allow large rotations while the substrate is modeled by small strain elasticity. Indeed, the considered instabilities are governed by nonlinear geometric effects for the stiff material, while the effects are much smaller for the soft material. For rectangular film/substrate systems, a thorough investigation on comparison between finite strain hyperelastic model and small strain elastic model with respect to a wide range of Young's modulus was carried out in [32]. It demonstrates that unless the stiffness ratio is rather small, e.g. $E_{f} / E_{s} \approx \mathcal{O}(10)$, deformation of the system can be so large that finite strain constitutive laws have to be taken into account. In most cases of film/substrate systems, i.e. $E_{f} / E_{s} \gg \mathcal{O}(10)$, small strain elastic models appear to be sufficient and are qualitatively or even quantitatively equivalent to finite strain hyperelastic models. This remains valid for trapezoidal film/substrate structures where macroscopic strain normally does not exceed $4 \%$ and thus remains in linear regime [29]. Therefore, we consider in the following Hookean elasticity for the potential energy of the system for reasons of simplicity.

The following computational technique has been chosen to avoid most restrictive assumptions on initial geometric imperfections that are generally performed in the literature [21,22]: the considered domain herein is three-dimensional without geometric imperfection and pathfollowing technique provides the post-buckling response of the system beyond the primary bifurcation [23]. On the bottom surface of the substrate, deflection $w$ and tangential traction are taken to be zero. In this paper, force load is applied to the film whereas the substrate is load free. Common material characteristics and geometric properties of the tapered film/substrate system are shown in Table 1, which are the same as the ones in [23,24]. Different dimensional parameters for each case are presented in Table 2, where two classes of geometry, 

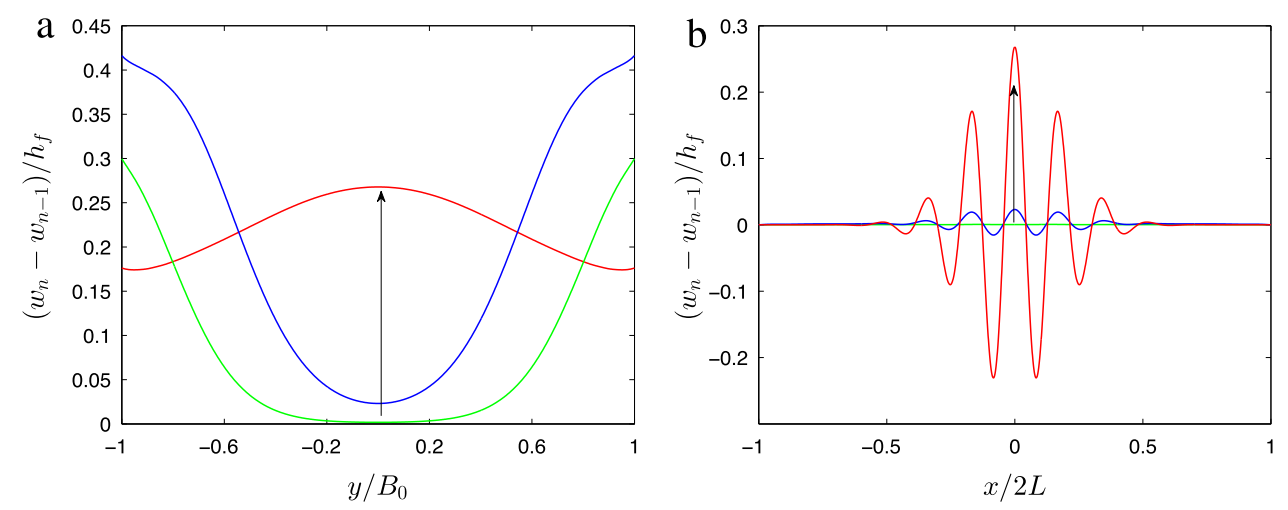

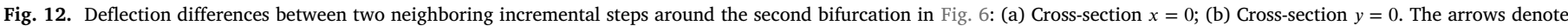
the evolution of incremental direction under increasing load.
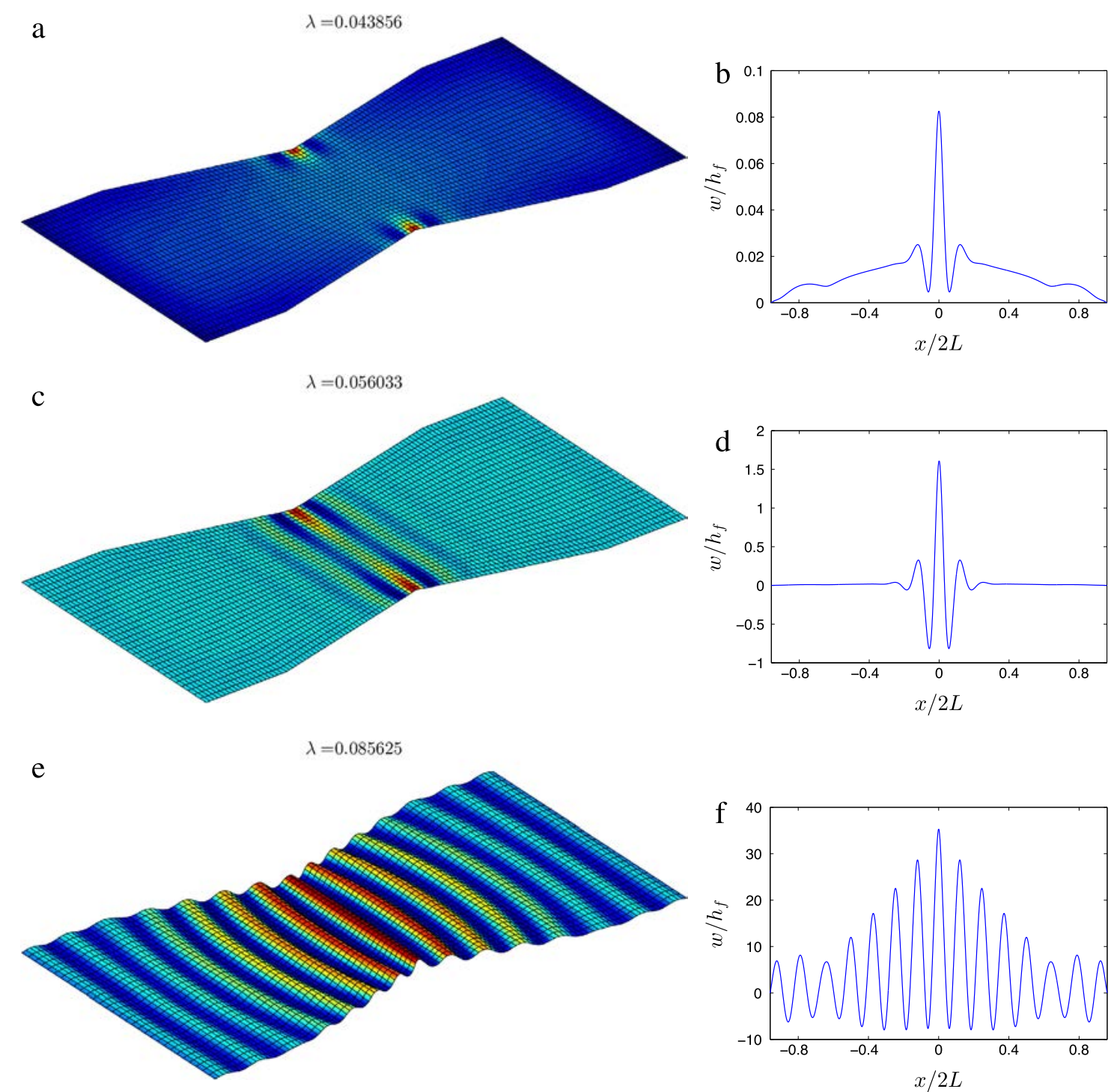

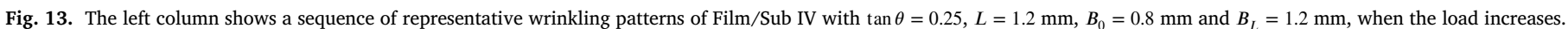
The color contour represents $w / h_{f}$. The right column presents the corresponding wrinkling modes along the length.

i.e. trapezoid and trapezoid-rectangle, are taken into account (see Fig. 5). Huge ratio of Young's modulus, $E_{f} / E_{s}$, determining the critical wavelength $\ell_{c r}$ as shown in Eq. (3), is considered. Compliant materials for the substrate, such as elastomers, are nearly incompressible with Poisson's ratio $v_{s}=0.48$. In order to trigger a transition from the fundamental to the bifurcated branch, a small perturbation force, $f_{z}=$ $10^{-8}$, is imposed on the shell. Introduction of such small perturbation forces is a common technique for solving bifurcation problems by continuation techniques [38,39], even when using commercial finite element codes. This artifice could be avoided by applying a specific 

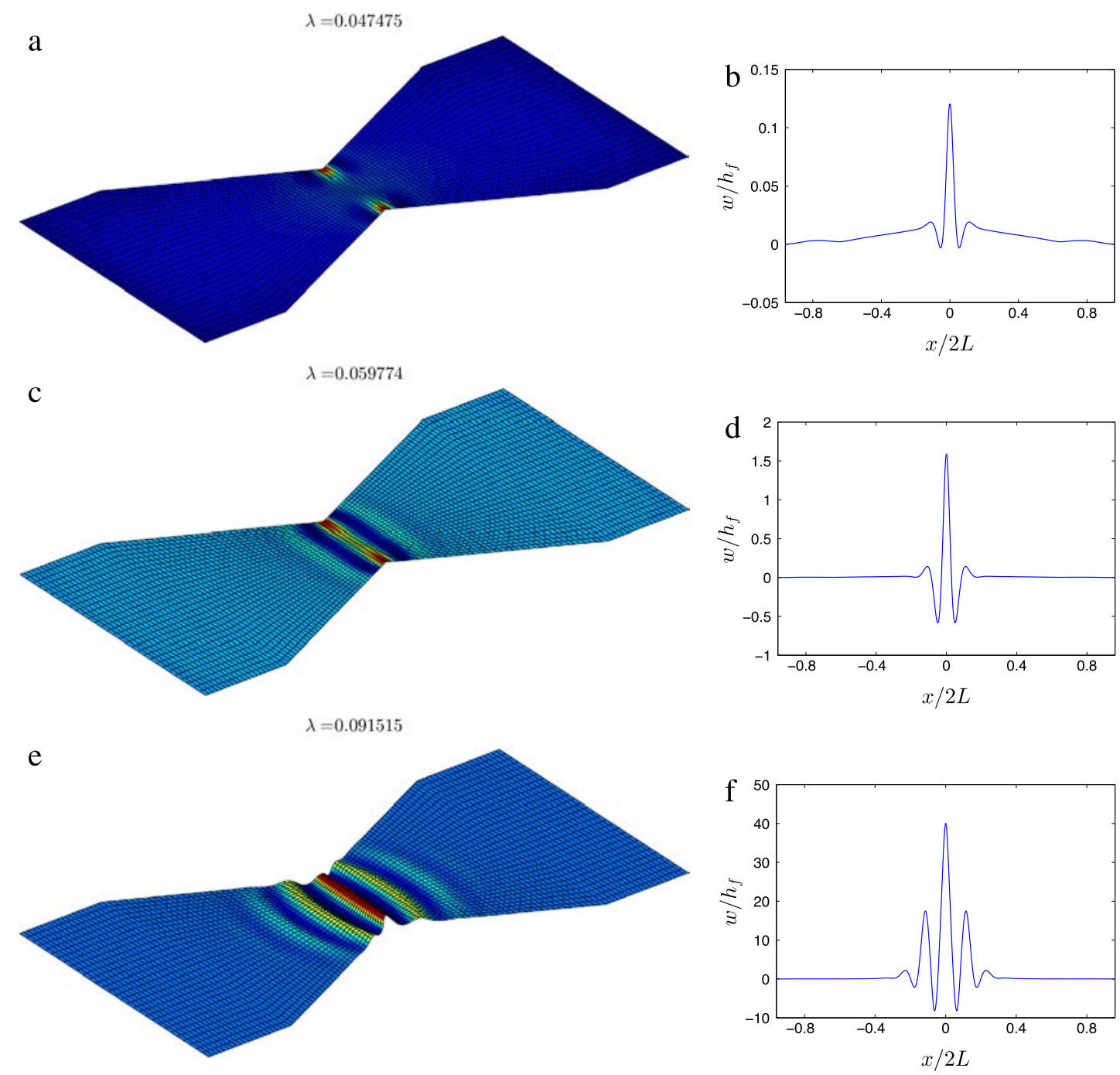

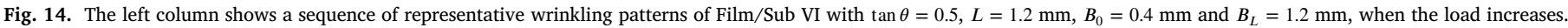
The color contour represents $w / h_{f}$. The right column presents the corresponding wrinkling modes along the length.

procedure to compute the bifurcation branch as in [40,41]. In this paper, the perturbation force $f_{z}$ allows computing the whole bifurcated branch with a single continuation algorithm. The number of elements required for a convergent solution was carefully examined. Bifurcation points are detected by the criterion of small step accumulation. Indeed, when the starting point of a step is close to bifurcation, the radius of convergence of Taylor series coincides with the distance to the singular point, which explains that the continuation process 'knocks' against the bifurcation [42]. More advanced techniques are available for bifurcation detection such as bifurcation indicator [23,24] and power series analysis [43]. In what follows, we will explore the spatial formation and evolution of irregular wrinkling patterns.

\subsection{Trapezoid}

First, we investigate pattern formation and post-buckling evolution via Film/Sub I with $\theta=0.25$ under uniaxial compression along $x$ direction. Clamped boundary conditions are applied on side $B_{L}$ and symmetry condition is considered on side $B_{0}$. The two taper edges are set free. Consequently, the surface layer is meshed with $70 \times 30$ shell elements to ensure at least six quadratic elements within a single wave. The substrate is compatibly discretized by 10500 solid elements with five layers. Mesh convergence was carefully examined. Totally, the film/substrate system contains 85227 degrees of freedom (DOF) including the Lagrange multipliers.
The critical load of graded sinusoidal wrinkles based on the linearized stability analysis of 1D beam/foundation model is determined by Eq. (3). By introducing the material and geometric parameters of Film/Sub I listed in Tables 1 and 2, one can obtain analytical solution of critical compressive force $F_{c r} / B_{L}=0.051 \mathrm{~N} / \mathrm{mm}$, which agrees well with our 3D finite element results (about $0.046 \mathrm{~N} / \mathrm{mm}$ for the first bifurcation in Fig. 6). Two representative wrinkling patterns on the supercritical load-displacement curve are illustrated in Fig. 7. A localized corner mode occurs on the shorter edges at the critical load, which corresponds to the region where compressive stress is larger. This localized mode is captured by the 3D finite element model [23] incorporating the continuation technique ANM, while it has not been observed through linearized eigenvalue analysis [29]. When load increases to the second bifurcation, localized corner pattern tends to be a graded sinusoidal shape where the amplitude fades along $x$ direction. This wavy pattern grows and spreads along the length beyond the second bifurcation, while the wavelength remains almost constant. These irregular wrinkles are mainly caused by the tapered geometric gradient that leads to stress gradient in the film.

The second case is dedicated to Film/Sub II with a smaller $\theta=0.15$. Half of the system is taken into account for calculation due to symmetry. The mesh, load and boundary conditions are the same as before. Similar supercritical bifurcation diagram as in Film/Sub I is found, except a slight change of critical load $\left(0.049 \mathrm{~N} / \mathrm{mm}\right.$ in Fig. 8 and $F_{c r} / B_{L}=0.050$ 
$\mathrm{N} / \mathrm{mm}$ for analytical solution). A sequence of representative wrinkling patterns on the post-buckling curve is shown in Fig. 9. The same bifurcation scenario is observed in the case of Film/Sub III with a shorter $B_{0}$ (see Fig. 10). Therefore, a generic mechanical response emerges for any trapezoidal film/substrate system: a supercritical, stable postbifurcation and a ribbed buckling shape localized on the shorter side at the beginning which then tends to be a graded wavy pattern.

A top view of the final deformed shape for each case is depicted in Fig. 11. One can observe that wavelength varies from the cases, which implies the influence of trapezoidal topology as explained in the last terms on the right-hand sides of Eq. (3). Furthermore, spatially graded wrinkles are not straightly ribbed along $y$ direction but have a wavy curvature perpendicular to the taper edges, indicating that the wave direction varies as well. This is mainly due to stress relaxation along the edges and is actually distinguished from the analytical assumption on 1D sinusoidal wrinkles in models (4) and (7). Note that such orthogonality between the boundary and instability patterns seems a generic feature, which have been previously observed in experimental fluid mechanics [34].

Competition between plate-like and beam-like post-buckling responses can be observed in Figs. 6, 8 and 10, where plate-like stiffening behavior appears between two bifurcations, while beam-like softening behavior occurs beyond the second bifurcation. Note that the second bifurcation can be identified from the considerable deflection variations (instability mode change) between two neighboring incremental steps around the point accumulation region (see Fig. 12 for example). Before the second bifurcation, the instability mode is completely localized near the corners, while it develops mainly in the center after the bifurcation. One can see that the range of plate-like response shortens when the tapered angle decreases. This means for a sharp taper, platelike response dominates the post-buckling behavior, while for a small tapered angle, beam-like response is more significant.

\subsection{Trapezoid-rectangle}

To further explore the effect of tapered angle on irregular wrinkling pattern and its evolution, we consider trapezoid-rectangle geometry with finite taper dimension, i.e. the cases of Film/Sub IV-VII. The symmetry, mesh, loading and boundary conditions remain the same as before. Results on pattern evolution are respectively reported in Figs. 13 and 14, and a top view of the final pattern shape for each case is presented in Fig. 15. It can be seen that inside the taper region, wavy patterns emerge; while outside the taper region, straight stripes appear. The transition between the two happens at the interface where the wavelength and its amplitude alter. Nevertheless, for the cases with a relative large geometric gradient, wavy patterns are constrained inside the taper region and cannot propagate outside (see Figs. 14, 15(c) and (d)).

\section{Concluding remarks}

Post-buckling evolution of irregular wrinkling patterns in trapezoidal film/substrate systems was investigated from a quantitative point of view. Numerical simulations were performed based on a 3D finite element model, associating geometrically nonlinear shell formulation for the surface layer and linear elasticity for the substrate. This model can describe moderately large rotations and displacements on the trapezoidal surface. The resulting nonlinear differential equations were solved by a robust path-following continuation technique, namely ANM, which is able to detect secondary bifurcations on a severely nonlinear response curve and to trace the whole post-wrinkling evolution on the equilibrium path. Occurrence of a localized corner mode and evolution of fading wrinkles have been captured. These ribbed and graded patterns are caused by stress gradient stemming from irregular geometric topology. In fact, graded wrinkles are not straight stripes but hold a wavy curvature shape perpendicular to the trapezoidal edges due to the
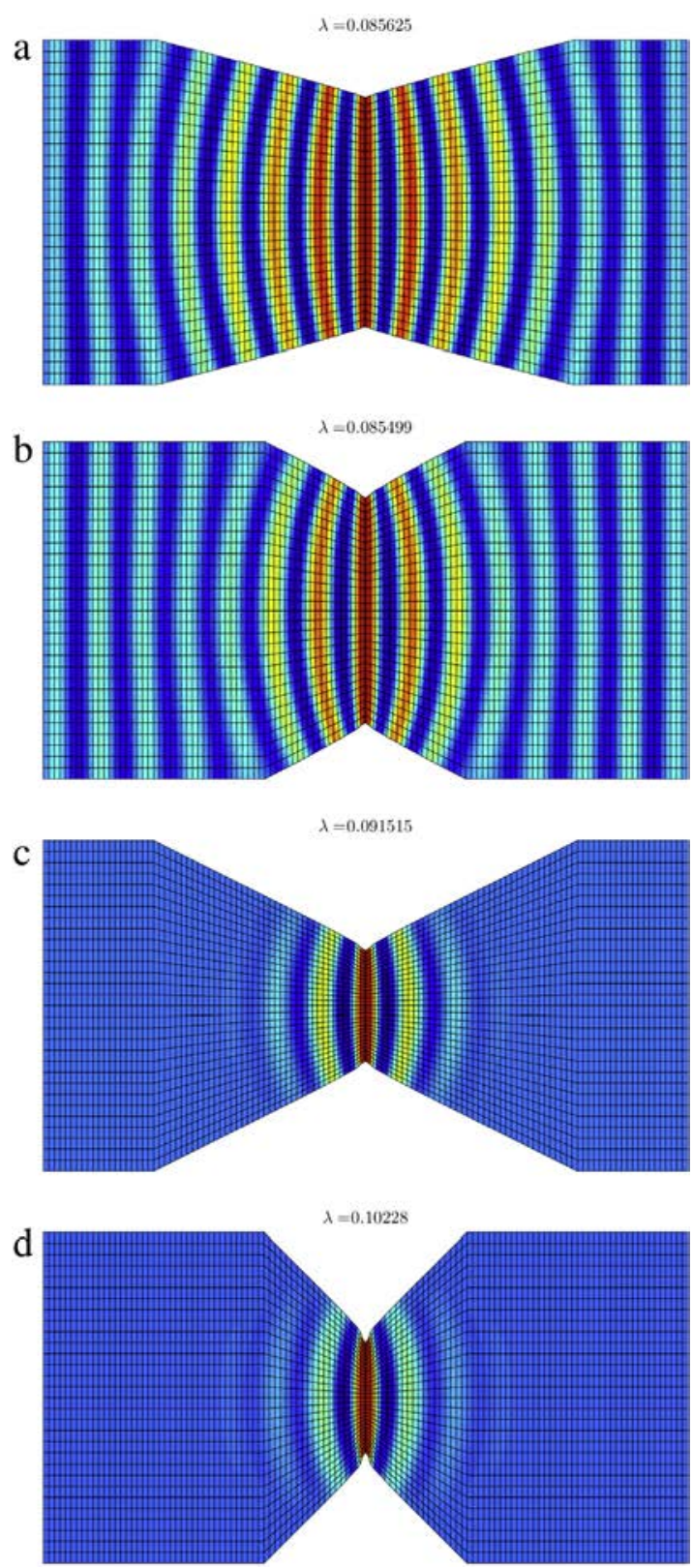

Fig. 15. Top view of final patterns: (a) Film/Sub IV; (b) Film/Sub V; (c) Film/Sub VI; (d) Film/Sub VII. The color contour denotes $w / h_{f}$.

release of stresses on the boundary, and this phenomenon cannot be captured by $1 \mathrm{D}$ beam/foundation models. Besides, competition between plate-like and beam-like post-wrinkling responses has been discussed. The significance of plate-like behavior increases with the raising of tapered angle, and vice versa. The numerical results could be helpful for the design of film/substrate bilayers to achieve desired irregular wavy wrinkling patterns, for example, for potential applications of fabricating hierarchical morphology holding special hydrodynamic or aerodynamic properties such as the reduction of turbulent drag/hydrodynamic friction as found in sharkskin and dragonfly wings $[1,44]$.

\section{Acknowledgments}

This work is supported by the National Natural Science Foundation of China (Grant Nos. 11602058, 11461161008, 11772094), Shanghai Education Development Foundation and Shanghai Municipal Education 
Commission (Shanghai Chenguang Program, Grant No. 16CG01), startup fund from Fudan University, and National Key Research and Development Program of China (2016YFB0700103). FX and MPF are also grateful for the financial support from the French National Research Agency ANR (LabEx DAMAS, Grant No. ANR-11-LABX-0008-01).

\section{References}

[1] K. Efimenko, M. Rackaitis, E. Manias, A. Vaziri, L. Mahadevan, J. Genzer, Nested self-similar wrinkling patterns in skins, Nature Mater. 4 (2005) 293-297.

[2] J. Yin, X. Chen, I. Sheinman, Anisotropic buckling patterns in spheroidal film/substrate systems and their implications in some natural and biological systems, J. Mech. Phys. Solids 57 (2009) 1470-1484.

[3] H.H. Dai, Y. Liu, Critical thickness ratio for buckled and wrinkled fruits and vegetables, EPL (Europhys. Lett.) 108 (2014) 44003-1-44003-6.

[4] S. Budday, C. Raybaud, E. Kuhl, A mechanical model predicts morphological abnormalities in the developing human brain, Sci. Rep. 4 (2014) 5644-1-5644-7.

[5] Q. Wang, X. Zhao, A three-dimensional phase diagram of growth-induced surface instabilities, Sci. Rep. 8 (2015) 8887-1-8887-10.

[6] C. Zhang, B. Li, X. Huang, Y. Ni, X.Q. Feng, Morphomechanics of bacterial biofilms undergoing anisotropic differential growth, Appl. Phys. Lett. 109 (2016) 143701-1143701-5.

[7] P. Sáez, A.M. Zöllner, Mechanics reveals the biological trigger in wrinkly fingers, Ann. Biomed. Eng. 45 (2017) 1039-1047.

[8] N. Bowden, S. Brittain, A.G. Evans, J.W. Hutchinson, G.M. Whitesides, Spontaneous formation of ordered structures in thin films of metals supported on an elastomeric polymer, Nature 393 (1998) 146-149.

[9] F. Brau, H. Vandeparre, A. Sabbah, C. Poulard, A. Boudaoud, P. Damman, Multiplelength-scale elastic instability mimics parametric resonance of nonlinear oscillators, Nat. Phys. 7 (2011) 56-60.

[10] J.A. Rogers, T. Someya, Y. Huang, Materials and mechanics for stretchable electronics, Science 327 (2010) 1603-1607.

[11] Y. Li, Reversible wrinkles of monolayer graphene on a polymer substrate: toward stretchable and flexible electronics, Soft Matter 12 (2016) 3202-3213.

[12] E.P. Chan, A.J. Crosby, Fabricating microlens arrays by surface wrinkling, Adv. Mater. 18 (2006) 3238-3242.

[13] D. Breid, A.J. Crosby, Curvature-controlled wrinkle morphologies, Soft Matter 9 (2013) 3624-3630.

[14] D. Terwagne, M. Brojan, P.M. Reis, Smart morphable surfaces for aerodynamic drag control, Adv. Mater. 26 (2014) 6608-6611.

[15] J.A. Howarter, C.M. Stafford, Instabilities as a measurement tool for soft materials, Soft Matter 6 (2010) 5661-5666.

[16] S. Zeng, R. Li, S.G. Freire, V.M.M. Garbellotto, E.Y. Huang, A.T. Smith, C. Hu, W.R.T. Tait, Z. Bian, G. Zheng, D. Zhang, L. Sun, Moisture-responsive wrinkling surfaces with tunable dynamics, Adv. Mater. 29 (2017) 1700828-1-1700828-7.

[17] C. Zong, Y. Zhao, H. Ji, X. Han, J. Xie, J. Wang, Y. Cao, S. Jiang, C. Lu, Tuning and erasing surface wrinkles by reversible visible-light-induced photoisomerization, Angew. Chem. Int. Ed. 55 (2016) 1-6.

[18] X. Chen, J.W. Hutchinson, Herringbone buckling patterns of compressed thin films on compliant substrates, J. Appl. Mech. 71 (2004) 597-603.

[19] Z.Y. Huang, W. Hong, Z. Suo, Nonlinear analyses of wrinkles in a film bonded to a compliant substrate, J. Mech. Phys. Solids 53 (2005) 2101-2118.

[20] B. Audoly, A. Boudaoud, Buckling of a stiff film bound to a compliant substratePart I: formulation, linear stability of cylindrical patterns, secondary bifurcations, J. Mech. Phys. Solids 56 (2008) 2401-2421.
[21] S. Cai, D. Breid, A.J. Crosby, Z. Suo, J.W. Hutchinson, Periodic patterns and energy states of buckled films on compliant substrates, J. Mech. Phys. Solids 59 (2011) 1094-1114.

[22] Y. Cao, J.W. Hutchinson, Wrinkling phenomena in neo-Hookean film/substrate bilayers, J. Appl. Mech. 79 (2012) 031019-1-031019-9.

[23] F. Xu, M. Potier-Ferry, S. Belouettar, Y. Cong, 3D finite element modeling for instabilities in thin films on soft substrates, Int. J. Solids Struct. 51 (2014b) 36193632.

[24] F. Xu, M. Potier-Ferry, S. Belouettar, H. Hu, Multiple bifurcations in wrinkling analysis of thin films on compliant substrates, Int. J. Nonlinear Mech. 76 (2015b) 203-222.

[25] Y. Fu, Z. Cai, An asymptotic analysis of the period-doubling secondary bifurcation in a film/substrate bilayer, SIAM J. Appl. Math. 75 (2015) 2381-2395.

[26] L. Zhuo, Y. Zhang, The mode-coupling of a stiff film/compliant substrate system in the post-buckling range, Int. J. Solids Struct. 53 (2015) 28-37.

[27] C.G. Wang, Y.P. Liu, H.F. Tan, Global and local interactive buckling behavior of a stiff film/compliant substrate system, Int. J. Solids Struct 102-103 (2016) 176-185.

[28] F. Xu, M. Potier-Ferry, A multi-scale modeling framework for instabilities of film/substrate systems., J. Mech. Phys. Solids 86 (2016) 150-172.

[29] S. Raayai-Ardakani, J.L. Yagüe, K.K. Gleason, M.C. Boyce, Mechanics of graded wrinkling, J. Appl. Mech. 83 (2016) 121011-1-121011-10.

[30] F. Xu, H. Hu, M. Potier-Ferry, S. Belouettar, Bridging techniques in a multi-scale modeling of pattern formation, Int. J. Solids Struct. 51 (2014a) 3119-3134.

[31] B. Cochelin, N. Damil, M. Potier-Ferry, Méthode Asymptotique Numérique, Hermès Science Publications, Paris, 2007.

[32] F. Xu, Y. Koutsawa, M. Potier-Ferry, S. Belouettar, Instabilities in thin films on hyperelastic substrates by 3D finite elements, Int. J. Solids Struct 69-70 (2015a) $71-85$.

[33] A. Javili, B. Dortdivanlioglu, E. Kuhl, C. Linder, Computational aspects of growthinduced instabilities through eigenvalue analysis, Comput. Mech. 56 (2015) 405420.

[34] J.E. Wesfreid, S. Zaleski, in: Cellular Structures in Instabilities. Lecture Notes in Physics, Vol. 210, Springer-Verlag, Heidelberg, 1984.

[35] N. Damil, M. Potier-Ferry, Influence of local wrinkling on membrane behaviour: A new approach by the technique of slowly variable Fourier coefficients, J. Mech. Phys. Solids 58 (2010) 1139-1153.

[36] K. Mhada, B. Braikat, H. Hu, N. Damil, M. Potier-Ferry, About macroscopic models of instability pattern formation, Int. J. Solids Struct. 49 (2012) 2978-2989.

[37] N. Damil, M. Potier-Ferry, H. Hu, Membrane wrinkling revisited from a multi-scale point of view, Adv. Model. Simul. Eng. Sci. 1 (6) (2014).

[38] E. Doedel, AUTO: A program for the automatic bifurcation analysis of autonomous systems, Congr. Numer. 30 (1981) 265-284.

[39] E.L. Allgower, K. Georg, Numerical Continuation Methods, Springer-Verlag, Berlin, 1990.

[40] E.H. Boutyour, H. Zahrouni, M. Potier-Ferry, M. Boudi, Bifurcation points and bifurcated branches by an asymptotic numerical method and Padé approximants, Internat. J. Numer. Methods Engrg. 60 (2004) 1987-2012.

[41] P. Vannucci, B. Cochelin, N. Damil, M. Potier-Ferry, An asymptotic-numerical method to compute bifurcating branches, Internat. J. Numer. Methods Engrg. 41 (1998) 1365-1389.

[42] S. Baguet, B. Cochelin, On the behaviour of the ANM continuation in the presence of bifurcations, Commun. Numer. Methods. Eng. 19 (2003) 459-471.

[43] B. Cochelin, M. Medale, Power series analysis as a major breakthrough to improve the efficiency of Asymptotic Numerical Method in the vicinity of bifurcations, J. Comput. Phys. 236 (2013) 594-607.

[44] A. Schweikart, A. Fery, Controlled wrinkling as a novel method for the fabrication of patterned surfaces, Microchim. Acta 165 (2009) 249-263. 